\title{
Effective expediting to improve project due date and cost performance through buffer management
}

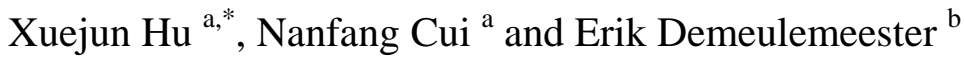 \\ ${ }^{a}$ School of Management, Huazhong University of Science and Technology, Wuhan, China \\ ${ }^{\mathrm{b}}$ Department of Decision Sciences and Information Management, KU Leuven, Belgium
}

The rapidly changing marketplace together with the increasing complexity of contemporary projects makes it more likely that project activities will have uncertain durations, incurring a generally low probability of on-time delivery. Thus, project control that aims to track the project performance and to expedite relevant activities when necessary has become the main aspect within the scope of project management in order to ensure a successful scheduling outcome. The Critical Chain Scheduling and Buffer Management (CC/BM) has shown to provide a popular approach to build robust project schedules and to offer a valuable control tool for coping with schedule variability. However, the most current buffer management practice faces a problem of neglecting the cost information when taking expediting actions. In view of this defect, we introduce a new control procedure on the basis of $\mathrm{CC} / \mathrm{BM}$ that evaluates the probability of successful project completion relative to the cost of crashing and that determines when to expedite which activities in a cost-effective manner. Results of an experimental application of the proposed method present its relative dominance over the currently widely adopted buffer management approach with respect to project time and cost performance.

Key words: Project control; Critical chain buffer management; Activity expediting; Cost performance; On-time delivery

\section{Introduction}

Goldratt’s Critical Chain Scheduling and Buffer Management (CC/BM) methodology, which is a practical translation of the theory of constraints to project management, has 
been extensively explored in the academic world (Demeulemeester and Herroelen, 2002; Ricketts, 2008; Rabbani et al., 2007; Vanhoucke, 2012; Peng and Huang, 2014;Tian and Demeulemeester, 2014). Meanwhile, CC/BM has found its way towards practical applications in production systems in the manufacturing and service industries. As a matter of fact, 30\% of global economic activities are arranged and performed in a project way according to some statistics (Turner, 2008). Practical examples abound in the job lot production, the new product development, the engineer-to-order manufacturing, etc. In these cases, highly customized products are required to be designed and delivered in detail following the specifications in the orders placed by customers (Zhu and Pandit, 2007). It makes sense that each order is handled as a project since an order includes several processes and a project involves unique sets of activities/tasks, and that a project management method is generally applicable (Alfieri et al., 2011; Bie et al., 2012). As such, CC/BM can be used to build robust project schedules or production plans that is robust against uncertain disruptions by inserting various types of buffers and to improve the system's performance to meet the due date through buffer management (BM).

In $\mathrm{CC} / \mathrm{BM}$, the critical chain is defined as the longest path which not only considers the precedence relations, but also takes into account the resource dependencies (Herroelen and Leus, 2001). There are two types of time buffers in the single project environment: a project buffer (PB) and feeding buffers (FBs). A PB is positioned at the end of the critical chain to protect due-date performance from variations in the critical activities. FBs are placed whenever a non-critical task joins the critical chain in order to protect the critical chain from potential delays caused by the feeding paths (Bie et al., 2012). In addition to providing aggregated uncertainty protection, buffers are supposed to act as essential mechanisms to monitor schedule deviations and to take appropriate actions in the project implementation phase. Thus, buffer management (BM) is the key to tracking and controlling the project performance.

We note that an effective project control method should resolve the following three basic issues: (1) when are corrective actions necessary? (2) which activity (or 
combination of activities) should the management select to expedite? (3) to what extent shall managers contain activity durations in order to effectively shorten the project completion time at minimum cost? While the current BM framework offers a great solution to the first issue, it fails to provide more explicit guidelines concerning which activities to expedite and to what extent. Besides, the current BM model is over-simplistically based on experience without cost considerations. Therefore, we seek to supplement the conceptual guidance to project time and cost control within the framework of CC/BM. To this aim, an algorithm is presented that makes use of a pre-defined simulation-based index in order to timely bring the late running project partly back on track while keeping a low crash cost. Our computational studies show that, relative to the previous BM approach, our expediting/crashing policies provide a higher level of performance with respect to both time and cost.

The remainder of this paper is divided into four sections to help motivate, illustrate and evaluate the suggested method. Section 2 provides a review of the literature that is relevant to this research. In Section 3 the primary buffer management system is briefly described, and then the new BM-based expediting approach considering the cost is introduced. Section 4 presents a simulation study to test the performance of the proposed method. In the last section, we summarize this research and identify some ideas for future work.

\section{Literature review}

In today's highly competitive and rapidly changing marketplace, the execution of a project schedule is increasingly faced with various uncertainties that may lead to numerous schedule disruptions, increasing the risk of failing to meet completion deadlines (Herroelen and Leus, 2004; Wang, 2005).One alternative for completing projects on schedule is the expediting or crashing of activity durations, e.g., through the use of highly-skilled labor or additional resources, the substitution of equipment, the improvement of raw material or the adoption of new technology, etc. This inevitably results in a financial trade-off between the project completion time and the cost of expediting (Klastorin2004, Kerzner 2013). As such, project time and cost 
control has become one of the biggest concerns in order to achieve single or multiple project objectives.

The primary practice for activity crashing under the deterministic Critical Path Method (CPM) is the period-by-period least marginal cost approach developed by Kelley (1961) and Fulkerson (1961). Over the years, many authors (e.g., Phillips and Dessouky, 1977; Baker, 1997;Gutjahret al., 2000; Mitchell and Klastorin, 2007; Jiang and Zhu, 2010; Chen and Tsai, 2011; etc.) have come up with various methods of improvements or alternatives to solving the time-cost trade-off problem (or the project compression/expediting/crashing problem) under deterministic as well as stochastic conditions in the project planning phase. The studies that help to dynamically determine which activities to expedite in the project implementation phase, however, are very scarce. Johnson and Schou (1990) attempted to modify the criticality index (CI, Van Slyke, 1963) by combining it with the expediting cost per unit time to generate a new criterion in the hope of providing a better crashing decision support. Bowman(2006) presented a new structure for effective project control, which involves specifying a specification limit for the duration of each activity on the purpose of achieving targeted timely project completion probabilities (further referred to as TPCPs, Van de Vonder et al., 2005) at minimum cost, and then management actions should be taken in case the specification limit is exceeded during project execution. Bregman (2009a) introduceda matrix simulation approach for solving the dynamic probabilistic expediting problem in order to satisfy a predefined TPCP while minimizing the expediting cost.

The papers reviewed above are mostly based on the CPM or Program Evaluation and Review Technique (PERT) analyses, both of which have long been questioned by many scholars (Goldratt, 1997; Herroelen and Leus, 2005; Blackstone et al., 2009; Kerzner, 2013; Goh and Hall, 2013). The criticisms include the inadequate estimation of task durations, the ignorance of human behavior (e.g., the student syndrome, Parkinson's Law, multitasking), the elimination of resource contention, etc. Hence, crashing policies based on the traditional project scheduling techniques are very likely to result in poor action decisions and project performance, for example, when 
activities have uncertain durations or projects have multiple critical (or near-critical) paths (Bregman, 2009b). As a new methodology for comprehensive project management considering resource constraints and uncertainties in the project, CC/BM well addresses the above-mentioned issues. Specially, buffer management serves as a valuable control tool for expediting activities to keep the whole project on schedule.

The existing BM system mainly follows the practice established by Goldratt (1997) for production operations (Yuan et al., 2003; Wu et al., 2010; Turki et al., 2013; Büchmann-Slorup, 2014;Papadopoulos et al., 2013). That is, the buffer is equally divided into three regions and explicit action levels are set in terms of the buffer consumption (BC) (i.e. the "green-yellow-red" system). Herroelen and Leus (2001)further completed Goldratt's assertion by stating that BM provides a clear methodology to establish a feedback/warning mechanism to stimulate appropriate management actions. They also conducted simulation experiments to illustrate the effectiveness of the $\mathrm{BM}$ crashing mechanism in improving project schedule performance. Umble and Umble (2006) applied BM to health care systems by treating each patient as a new project; then the "green-yellow-red" system with unequal buffer zones was employed and significant improvements were generated in several application cases. Kuo et al. (2009) improved the overall due-date performance of wafer fabrication factories using aggregated time buffers according to Goldratt's monitoring idea. Leach (2005) questioned Goldratt's static monitoring notion,and he recommends the two buffer trigger lines to vary linearly over the planned duration of the project considering the fact that the uncertainty might decrease as the project progresses. We refer to this method as the Relative Buffer Management Approach (RBMA), with which we will conduct a simulation study to compare our method. A detailed explanation can be found in Section 3.1.

As can be seen from our overview, though application of buffer management has proliferated in recent years, the cost performance is generally not taken into account in the literature. Besides, we have seen no in-depth research with respect to which activities it will be important to control and to what extent in a project environment. Hence, there is a need for a more comprehensive project control approach (to 
effectively monitor schedule variations while maintaining a comparatively low crash cost) in projects with uncertain task durations, and the flaws of the different procedures noted above provided the motivation for this research.

\section{The proposed method for effective project expediting}

Although the predominant RBMA method has been successfully implemented by a growing number of companies, its model is not clearly described in the literature. In this section, we will first provide some basic terminologies and notations which are necessary for the understanding of subsequent analyses, and then the proposed BM-based expediting approach considering the cost is introduced.

\subsection{The basic CC/BM methodology}

For a project network that consists of zero-lag finish-start precedence constraints as well as resource dependencies, the critical chain (referred to as $h=1$ ) is identified by using some kind of accepted algorithm (see e.g., Tukel et al., 2006; Rabbani et al., 2007; Long and Ohsato, 2008; etc.) or software (e.g., ProChain, Project Development Institute, Scitor Corporation, etc.).Then the non-critical chains (referred to as $h=2,3, \mathrm{~L}, H)$ are determined and the project buffer as well as the feeding buffers are inserted. For more details about buffer sizing approaches, we refer to Tukel et al. (2006) and Bie et al. (2012).

The following definitions will be used throughout this paper:

$N$ : number of non-dummy activities

$H$ : number of chains

$n^{h}:$ number of activities on each chain

$I_{j}^{h}$ : the $j$ th activity on chain $h\left(1 \leq j \leq n^{h}, 1 \leq h \leq H\right)$

$B^{h}$ : the size of PB or FB for chain $h$

$D_{B}(i)$ : the baseline duration of activity $i$

$D_{R}(i)$ : the realized duration of activity $i$ (generated by using the Monte Carlo Simulation approach to simulate the project implementation process) 
$S(i)$ : the planned starting time of activity $i$

$s(i)$ : the realized starting time of activity $i$

During project execution managers keep track of buffer penetration with the completion of activities. By comparing the actual project implementation against the baseline schedule at time instant $t$ (see Figure 1), one can easily calculate how much of the buffer will be consumed (i.e. buffer consumption, $B C$ ) by the completion of activity $I_{j}^{h}$, as presented in the following formula:

$$
B C_{j}^{h}=\left(s\left(I_{j}^{h}\right)+D_{R}\left(I_{t j}^{h}\right)\right)-\left(S\left(I_{j}^{h}\right)+D_{B}\left(I_{j}^{h}\right)\right)
$$

This comparison gives the project manager an indication as to how well the project is progressing or the degree of deviations from the baseline schedule. Obviously, the buffer threshold values to trigger control actions vary as a function of chain completion. As such, the monitoring procedure of the RBMA method can be further illustrated by a so-called control chart in Figure 2 (Leach, 2005).

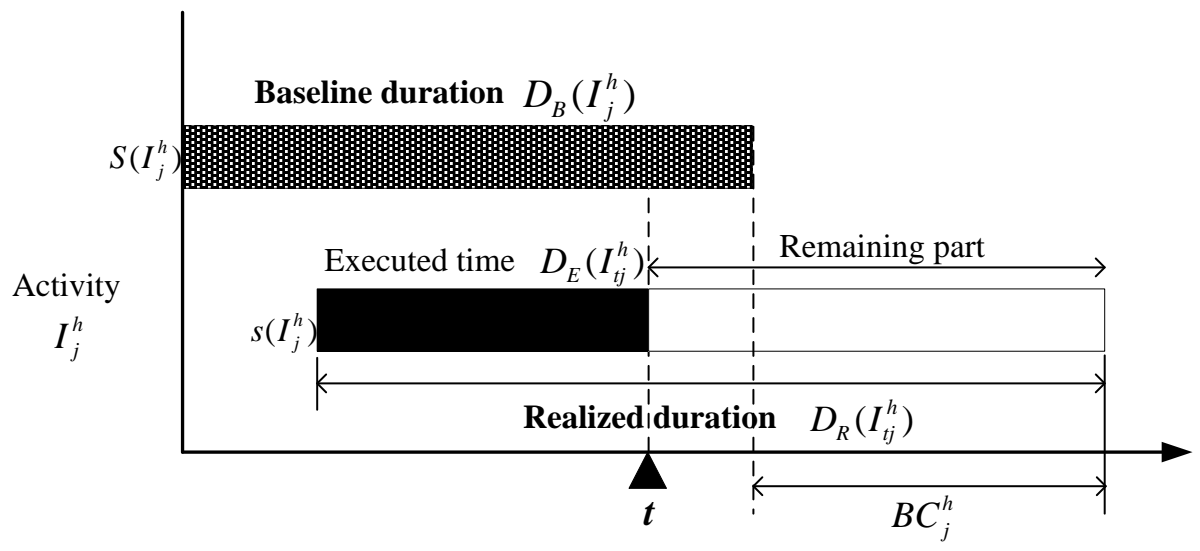

Figure 1. Activity execution status at time instant $t$ (project review) 


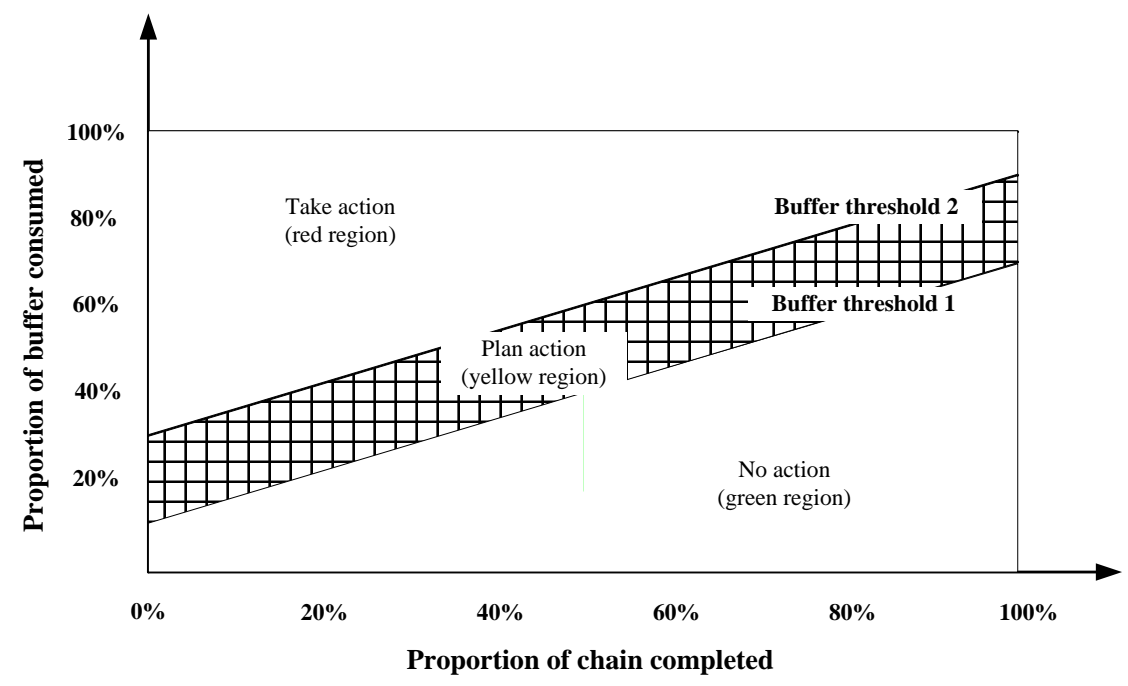

Figure 2. Buffer thresholds in the RBMA method

As can be seen from Figure 2, the vertical axis represents the proportion of the buffer consumed $(P B C)$, and the horizontal axis indicates the proportion of the chain completed (PCC):

$$
\begin{gathered}
P B C=B C_{j}^{h} / B^{h} \\
P C C=\left(\sum_{i=1}^{j} D_{B}\left(I_{i}^{h}\right) / \sum_{i=1}^{n_{h}} D_{B}\left(I_{i}^{h}\right)\right.
\end{gathered}
$$

We mathematically formulate the green-to-yellow transition (Buffer Threshold 1) and yellow-to-red transition (Buffer Threshold 2) as follows:

$$
\begin{aligned}
& \text { Buffer Threshold 1: } \quad P B C>a_{1} * P C C+b_{1} \\
& \text { Buffer Threshold 2: } \quad P B C>a_{2} * P C C+b_{2}
\end{aligned}
$$

In Equations (4) and (5), $a_{1}\left(a_{2}\right)$ represents the slope of the two buffer trigger lines and $b_{1}\left(b_{2}\right)$ is the intercept. The idea is that, if $\mathrm{BC}$ is negative or in the green region, there is no need to take action; if activity variation consumes the buffer to Threshold 1, project managers should plan actions for that chain to speed up the execution of the remaining tasks; if subsequent delays penetrate the buffer beyond Threshold 2, the management team should immediately take action to recover the buffer (Goldratt, 1997). Note that if the PB is consumed, the successive critical chain activities are made eligible for crashing; otherwise, the activities eligible to be 
expedited are the unfinished non-critical transitive predecessors of the penetrated FBs (Herroelen and Leus, 2001).This type of monitoring logic offers a reliable warning mechanism and informs the decision makers about what needs to be done in view of buffer penetration. Moreover, task priorities pertaining to resources can be set according to the type of consumed buffer (i.e. project buffer or feeding buffer) and the percentage that has been consumed. Therefore, by controlling the buffers, the project team will be more focused and the need for frequent rescheduling operations shall be reduced.

\subsection{Expediting a CC/BM schedule with cost considerations}

As reported earlier, the current buffer management practice illustrated above has a serious drawback of neglecting the expediting cost when managing the execution of a project. In addition, there has been no accepted rule concerning how to plan or act explicitly when buffer thresholds are triggered. These issues lie at the heart of this research.

\subsubsection{A mathematical model}

Before the new simulation-based algorithm is introduced, we present a mathematical model that aims to determine the best expediting option in situations where control action is necessary. We would like to point out that the model below is only aimed at the critical chain $(h=1)$ on which managerial diligence is needed, considering the fact that feeding buffers might provide sufficient protection on critical activities (the defect of this assumption will be discussed later).

$$
\begin{gathered}
\min \quad z=e c_{j}^{*} e t_{j}+\sum_{k}\left(e c_{k} * e t_{k}\right) \\
\text { s.t. } t+D_{R}(j)-D_{E}(j)+\sum_{k} \hat{d_{k q}}-\left(e t_{j}+\sum_{k} e t_{k}\right)-\delta_{q}^{+}+\delta_{q}^{-}=\delta, \\
k \in\left\{S_{j}^{1}\right\}, \quad s(j)<t \leq s(j)+D_{R}(j) \\
M * U_{q} \geq \delta_{q}^{+}, \quad q=1,2, \mathrm{~L}, m
\end{gathered}
$$




$$
\begin{gathered}
1-\frac{1}{m} \sum_{q=1}^{m} U_{q} \geq \alpha \\
e t_{i} \leq v_{i}, \quad i=1,2, \mathrm{~L}, N
\end{gathered}
$$

Activity set $\left\{S_{j}^{1}\right\}$ includes all the transitive successors of activity $j$ (the on-going activity at time instant $t$ ) on the critical chain that are eligible for future expediting.

$e c_{i}$ : the expediting cost per unit time for activity $i$. We use a linear cost-duration function in this research and hence $e c_{i}$ represents the marginal cost of reducing the processing time of activity $i$ by one unit.

$e t_{i}$ : the feasible expediting time for activity $i$

$m$ : the number of Monte Carlo simulation runs (index q)

$\hat{d_{i q}}$ : the estimated duration (subject to lognormal distribution in this paper) for each activity $i$ in replication $q$

$\delta$ : the project due date

$\delta_{q}^{+}$: the positive deviation from the due date $\delta$ (failure) in replication $q$

$\delta_{q}^{-}$: the negative deviation from the due date $\delta$ (success) in replication $q$

$U_{q}$ : a 0-1 variable denoting a failure for replication $q$

$\alpha$ : the predefined (required) TPCP threshold

$M$ :a large value (Big M Method)

$v_{i}$ : the maximum number of units that can be expedited for activity $i$.

It is practical to define a minimum crashed working time $\left(M I N D_{i}\right)$ for activity $i$ when control actions are involved. Although $M I N D_{i}$ could be any value, we just set it as a fixed percentage (Dper) of the baseline duration for each activity for the simplicity of the simulation study, i.e. $M I N D_{i}=\left\lceil\operatorname{Dper}^{*} D_{B}(i)\right\rceil$. Therefore, we have $v_{i}=\left(\hat{d}_{i}-M I N D_{i}\right)$

The objective of the above model (Equation (6)) is to determine the best 
expediting times for a set of tasks (on-going and future tasks) that satisfy a predefined TPCP requirement at minimum crash cost. The first constraint (Equation (7)) shows the relationship between activity crashing and the project due date $\delta$. Activity times can namely be reduced as a corrective action according to possible combinations of expediting options to meet the due date requirement. Equation (8) defines project due date performance as a 0-1 binomial outcome for all replications. Equation (9) displays a targeted probability of completion and Equation (10) sets a limit on the available expediting time for each activity.

At each project review the model can be solved to obtain the best crashing planbased on a certain activity duration distribution (e.g. beta or lognormal distribution).However, the actual execution of future activities might be so uncertain and dynamic that part of the pre-solved crashing plan will never be implemented. The start and end times of activities might fluctuate, influencing buffer penetration and action decisions accordingly. Furthermore, in reality it is not always the case that feeding buffers will fulfill their role as an adequate protection due to complex interactions among activities and frequent resource conflicts. In these cases the benefits of mathematical optimization will likely be lessened because of the probabilistic nature of the problem. Hence, we develop a simulation-based heuristic procedure that reflects the essence of the optimization model in order to provide a practical and beneficial expediting decision basis.

\subsubsection{The proposed solution procedure}

We use a Monte Carlo Simulation (MCS) approach to generate a random set of realizations of task durations. Many scholars have explored the use of MCS in network simulation studies, which takes into consideration the uncertainty/variability and which provides a reliable direction for the manager to enhance the actual execution of the project (Van Slyke, 1963; Creemers et al., 2014). The implementation of explicit control actions relies on what we define as the Expediting Efficiency Index(EEI). We start by simulating the project represented by a CC/BM baseline schedule a number of replications (i.e. $m$ ) without any control actions to get a 
$T P C P^{n o}$. We then artificially conduct a duration reduction for an individual activity (as an analogy to the expediting actions) by multiplying its realization times by a certain ratio ( Ratio) in the second simulation run, whereas the realization times of the other activities remain the same as in the former run. Thus, we have $e t_{j q}=\hat{d}_{j q}^{*}\left(1-\right.$ Ratio $\left._{q}\right), \quad q=1,2, \mathrm{~L}, m$. This enables us to obtain a TPCP ${ }_{j}^{\text {yes }}$ for activity $j$ for all replications. And the EEI of activity $j$ is formulated as follows:

$$
E E I_{j}=\frac{T P C P_{j}^{\text {yes }}-T P C P^{\text {no }}}{\overline{T E C_{j}}}, \quad j=1,2, \mathrm{~L}, N
$$

with $\overline{T E C_{j}}$ the average expediting cost of activity $j$ for all simulation runs. The function is $\overline{T E C_{j}}=\sum_{q=1}^{m} c\left(e t_{j q}\right) / m$, in which $c(\cdot)$ denotes the crash cost function (continuous or discrete) relative to the corresponding activity expediting times. Since we use a linear cost-duration function in this paper, $\overline{T E C_{j}}$ can be determined by the following formula:

$$
\overline{T E C_{j}}=e c_{j} * \overline{e t_{j}}=e c_{j} *\left(\sum_{q=1}^{m} e t_{j q} / m\right)
$$

Equation (11) estimates the expected benefit (change in the TPCP performance) by expediting activity $j$ with a certain amount of control work and associated costs. Namely, this index considers cost as a factor in identifying the effectiveness of crashing actions for each activity. It is suggested that expediting the activity with a higher value of EEI will be more effective to improve the estimated probability of meeting the project overall deadline. The fundamental idea of project control is then to give priority to the activities with higher EEI values when making corrective plans (i.e. when Threshold 1 is triggered). What we should pay attention to is the way that actual actions are taken. Specifically, decisions made at the planning stage directly determine the expediting time (and hence the completion time) of the next activity that is about to start, whereas the expediting times of future activities depend not only on the plan but also on subsequent re-evaluations of the real-time execution. In the 
RBMA, however, it is assumed that any buffer penetration of Threshold 2 (Equation

(5)) requires immediate action.

The algorithm can now be stated as follows:

1. Construct a buffered CC/BM schedule for a project (see Section 3.1).

2. Compute the EEI value of each activity according to Equation (11), and sort the values from high to low.

3. Initiate the project when $t=0$. The management can choose to evaluate the project and check buffer consumption when: (1) an activity or milestone event is completed; (2) a chain breaks off, or new activities are inserted. Or, managers can implement a monitoring policy dynamically on a real time basis or at the appropriate time intervals. We assume, in this paper, that the project will be evaluated each time an activity $\left(I_{j}^{h}\right)$ is completed.

4. At each project evaluation (time instant $t$ ), if it is checked that prolonged durations consume the buffer to Threshold 1 (Equation (4)), actions are preemptively planned or immediately taken according to the following rule:

a) Calculate Exceed $_{j}^{h}=B C_{j}^{h}-\left(a_{1}^{*} P C C+b_{1}\right) * B^{h} \quad$ (see Section 3.1, Equations (1)-(4)) for each chain $h=1,2, \mathrm{~L} H$.

b) Determine an activity set $\left\{S_{j}^{h}\right\}$ that includes all the transitive successors of activity $I_{j}^{h}$ on the same chain that are eligible for future expediting. Define another activity set $A=\varnothing$. Select an activity $k$ that satisfies $k \in\left\{S_{j}^{h}\right\}$ and $E E I_{k}=\max \left(E E I_{i}\right), \forall i \in\left\{S_{j}^{h} \backslash A\right\}$. Set $\tilde{e t}_{k}=\min \left(v_{k}\right.$, Exceed $\left._{j}^{h}\right)$, and update Exceed $_{j}^{h}=$ Exceed $_{j}^{h}-\tilde{e t}_{k}$. Then add activity $k$ into set $A$.

i. If $k$ happens to start at time instant $t+1$, set $e t_{k}=e \tilde{t}_{k}$. Then the actual completion time of activity $k$ should be equal to its simulated duration minus $e t_{k}$.

ii. Else, reset $e t_{k}=0$. 
c) If Exceed $_{j}^{h} \neq 0$, repeat the process of step 4(b). Otherwise, go to step 5.

5. Increase the review period $t=t+1$. Repeat the procedure of step 4 until the project is completed.

A more detailed illustration of the proposed project control procedure is outlined in Figure 3.

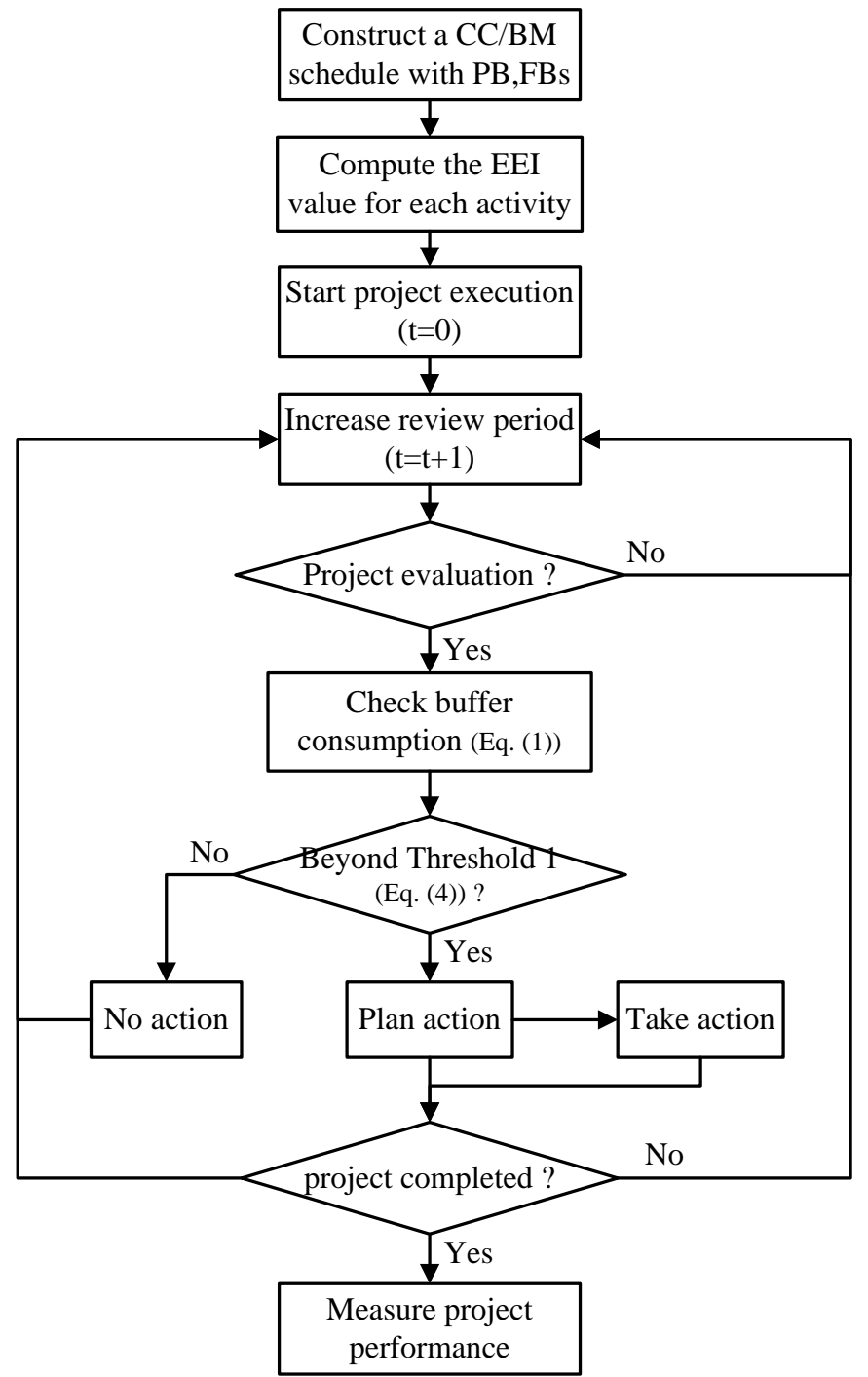

Figure 3. The proposed BM-based expediting procedure for project time and cost control

On the one hand, the EEI index uses stochastic task duration distributions together with the crash cost, thus enabling the best candidate activities to be determined. On the other hand, this approach allows project managers to exploit information concerning current schedule delays and expected probabilistic benefits to make expediting decisions preemptively, instead of waiting for the actual probabilistic 
outcomes. Therefore, we believe this to be a good model corresponding to actual project schedule-cost control decisions that would be practical and relatively easy to communicate and implement.

\section{Experimental tests of the proposed method}

To illustrate the capability of the proposed method (further referred to as Buffer Management Approach with Cost considerations, C-BMA) to select a good set of expediting options, a simulation study was performed to compare it with the RBMA method. In the RBMA, the expediting time for the next activity that will be subject to a correction action (i.e. when the buffer Threshold 2 is triggered) is assumed to be randomly generated from a uniform distribution over $\left[0, v_{k}\right]$. We do this in our simulation study because no explicit action guidelines have been specified in the literature.

\subsection{Experimental layout}

Our computational experimentation was conducted using the well-known PSPLIB data set (Kolisch and Sprecher, 1996) and the program was run on a personal computer in MATLAB language. To model the activity durations in the simulation, we use a right-skewed lognormal distribution with mean equal to the baseline duration. The standard deviation (i.e., sigma) represents various levels of project uncertainty. A lognormal distribution is also used by some other works (Herroelen and Leus, 2001; Tukel et al., 2006; Bie et al., 2012). For each project instance and for each value of sigma 1000 simulation replications were generated (i.e. $m=1000$ ). One thousand repetitions were deemed adequate since we have found no statistically significant differences when a larger number of simulation runs were tested. We set Dper $=50 \%$ in our experiment. Other values have been tested and have shown no significant differences in the management conclusions. Then Ratio is randomly generated from a uniform distribution over $[0.5,1]$ to model the crashed activity completion times.

The expediting cost per unit time $e c_{j}$ for activity $j$ is randomly generated from a 
uniform distribution over $[1,10]$ in each project. Then, before the actual execution of a project, an EEI value is calculated for each activity according to Equation (11)with sigma being generated from a uniform distribution over $[0,1]$ in each simulation run and $m=1000$. In this paper, we use the typical buffer threshold settings of $a_{1}=a_{2}=0.6, b_{1}=0.15, b_{2}=0.30$ as most CC/BM software does (Leach, 2005).

At the completion of each project, the average performance is measured for both buffer monitoring methods which consist of a comparison between the cost and effort the project manager has spent during the tracking process, and the effect of the corrective actions on project time performance.

To that purpose, the following performance indicators are defined:

- Number of expedited activities, EA. This is calculated as the sum of all activities that need to be expedited. This indicator measures the frequency with which management will act during project tracking. A larger EA means more activities are subject to corrective actions, increasing the complexity of project management as well as the probability of schedule change.

- Total expediting time, ET. This is calculated as the sum of the expediting times of all activities in each project. A longer ET means more control efforts are required to ensure a successful project completion. The function is $E T=\sum_{q=1}^{m}\left(\sum_{j} e t_{j q}\right) / m$.

- Total expediting costs, EC. EC $=\sum_{q=1}^{m}\left(\sum_{j} e c_{j} * e t_{j q}\right) / m$.

- Project completion time, PCT. This indicator refers to the simulated project completion time with or without threshold triggered corrective actions. The function is $P C T=\sum_{q=1}^{m} s_{N+1} / m$, with $N+1$ indicating the dummy end activity.

- Timely project completion probability, TPCP. This indicator refers to the probability that a project is completed within the projected due date (deadline) $\delta$. The function is $T P C P=\operatorname{Prob}\left(s_{N+1} \leq \delta\right)$.

In the simulation we determine the critical chain using the procedure of Tukel et 
al. (2006). The buffer sizes are calculated using theroot square error method (RSEM, Newbold, 1998), whose advantages are that it can utilize known task variation and will not generate very large or very small buffer sizes based on the length of the chain according to Tukel et al. (2006) and Bie et al. (2012). The remark has to be made that feeding buffers are inserted by treating them as extra activities without any consumption of resources. Then the buffered schedule is recomputed to obtain a precedence and resource feasible baseline schedule while maintaining the original critical chain. The project due date $\delta$ is then set equal to the maximum of the schedule length (i.e., the length of the critical chain) plus the project buffer size. As the readers may have noticed, apart from adding a project buffer, the management team can artificially set the final project deadline based on special requirements or simulation/analytical/statistical methods (Kuo et al., 2009). In this case, the time difference between the deadline and the length of the critical chain will be treated as the project buffer size since the buffer is also a kind of safety time. Throughout this paper, the execution of a $\mathrm{CC} / \mathrm{BM}$ schedule is done according to the roadrunner scheduling policy(Herroelen and Leus, 2001; Tian and Demeulemeester, 2014), favoring activities on the critical chain.

\subsection{Test results and analysis}

We first implemented both buffer monitoring methods on a single project network that is shown in Figure 4 (i.e. PSPLIB J301_1, which consists of 30non-dummy activities and consumes four kinds of resources with a constant availability of 12, 13, 4 and 12 units respectively). Table 1 displays the results. Then multiple instances of PSPLIB J30 project networks (with 30 activities) were tested and we report the statistical results in Table 2. Three levels of sigma (i.e., 0.3, 0.5 and 0.8) were used, representing a project uncertainty that is Low (L), Medium (M) or High (H), respectively. The results without any corrective actions were also listed for further comparison. Throughout the paper the baseline duration (50\% estimate) is assumed to be half of the safe estimate (90\%) as was described in most literature (see, e.g. Goldratt, 1997; Leach, 2005; Tukel et al., 2006; etc.) to calculate the buffer sizes. 


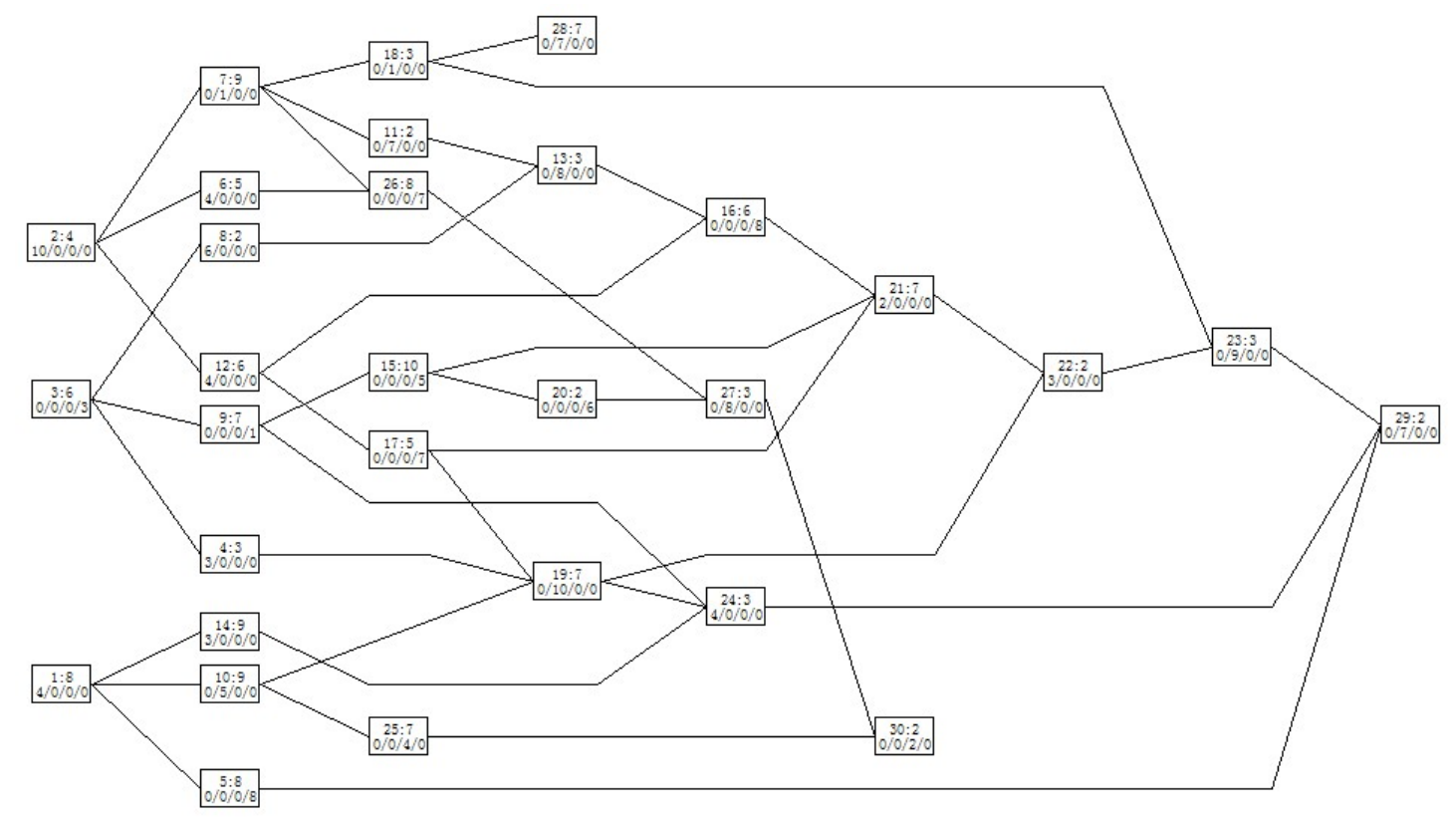

Figure 4. The project network of PSPLIB J301_1

Table 1. Performance comparison of both methods on PSPLIB J301_1

\begin{tabular}{|c|c|c|c|c|c|c|c|c|c|}
\hline \multirow{2}{*}{$\begin{array}{c}\text { Performance } \\
\text { indicator }\end{array}$} & \multicolumn{3}{|c|}{ Sigma $=0.3$} & \multicolumn{3}{|c|}{ Sigma $=0.5$} & \multicolumn{3}{|c|}{ Sigma $=0.8$} \\
\hline & C-BMA & RBMA & $\begin{array}{c}\text { No } \\
\text { action }\end{array}$ & C-BMA & RBMA & $\begin{array}{c}\text { No } \\
\text { action }\end{array}$ & C-BMA & RBMA & $\begin{array}{c}\text { No } \\
\text { action }\end{array}$ \\
\hline EA & 1.21 & 1.62 & & 1.27 & 1.61 & & 1.36 & 1.60 & \\
\hline ET & 1.82 & 3.39 & & 2.28 & 3.86 & & 2.95 & 5.13 & \\
\hline EC & 6.94 & 18.56 & & 8.27 & 20.08 & & 10.36 & 24.68 & \\
\hline ТРСР & 1 & 1 & 0.986 & 0.993 & 0.991 & 0.962 & 0.880 & 0.892 & 0.828 \\
\hline PCT & 60.91 & 60.53 & 62.35 & 61.48 & 61.24 & 63.46 & 66.34 & 66.01 & 69.08 \\
\hline
\end{tabular}

Table 2. Performance comparison of both methods on PSPLIB J30 project instances

\begin{tabular}{|c|c|c|c|c|c|c|c|c|c|}
\hline \multirow{2}{*}{$\begin{array}{c}\text { Performance } \\
\text { indicator }\end{array}$} & \multicolumn{3}{|c|}{ Sigma $=0.3$} & \multicolumn{3}{|c|}{ Sigma $=0.5$} & \multicolumn{3}{|c|}{ Sigma $=0.8$} \\
\hline & C-BMA & RBMA & $\begin{array}{c}\text { No } \\
\text { action }\end{array}$ & C-BMA & RBMA & $\begin{array}{c}\text { No } \\
\text { action }\end{array}$ & C-BMA & RBMA & $\begin{array}{c}\text { No } \\
\text { action }\end{array}$ \\
\hline EA & 0.47 & 0.52 & & 0.72 & 0.86 & & 0.98 & 1.13 & \\
\hline ET & 0.74 & 1.13 & & 1.19 & 1.96 & & 2.06 & 3.40 & \\
\hline EC & 3.55 & 6.12 & & 5.79 & 11.34 & & 10.26 & 23.34 & \\
\hline ТРСР & 1 & 1 & 0.997 & 0.996 & 0.995 & 0.951 & 0.944 & 0.941 & 0.886 \\
\hline PCT & 62.00 & 61.99 & 62.76 & 63.52 & 63.48 & 65.02 & 67.78 & 67.77 & 71.36 \\
\hline
\end{tabular}

As can be seen from Tables 1 and 2, the results of a single project example and those of large-scale implementations are consistent. In what follows, we will analyze the results in detail. 
The tables indicate that in both tracking methods as uncertainty in the project environment increases, the simulated project completion time, the total expediting time and the total expediting cost all increase, whereas the timely project completion probability declines as predicted. There is almost no change in the EA concerning the uncertainty level in a single project, whereas statistical results from multiple projects show the EA to be on the rise with an increase in sigma. It's obvious that the higher the uncertainty level, the more risks the project will be faced with. Consequently, the project is more likely to deviate from the baseline schedule, requiring a higher level of control efforts (time and cost) to meet the project deadline.

Note that both the C-BMA and the RBMA can guarantee higher TPCPs as well as shorter PCTs compared with that of the case where no control actions are involved (see the fourth and fifth row of Tables 1 and 2), indicating that buffer monitoring can be quite effective to ensure a successful project completion. Specially, sufficiently high levels of project on-time completion probabilities can be acquired even without corrective actions under low uncertainties, indicating the effectiveness of the CC/BM schedule itself in protecting the project due date.

Tables 1 and 2 also show that both methods generate approximately the same TPCPs and slightly different PCTs. However, the C-BMA is significantly more beneficial with respect to the total expediting times and the relevant crash costs under all uncertainty levels, and the performance gap between the two methods becomes more obvious for increasing values of sigma. Also, the number of expedited activities in the C-BMA method is lower than that of the RBMA. Due to the introduction of the EEI index which focuses on the cost effectiveness of activity crashing and helps to highlight management focus when corrective actions are necessary, the proposed method reduces management control efforts to a great extent. In general, more than half of the crash costs can be saved relative to the RBMA method.

Indeed, the proposed C-BMA procedure addresses the dynamic evaluation of a project's schedule performance as a whole and the selection of cost-effective activities in particular for crashing decisions. More precisely, the completion effectiveness and cost effectiveness for each activity are taken into consideration when making 
expediting plans and taking corrective actions. On the one hand, part of the planned action is delayed to those highly expediting-effective activities; on the other hand, the real-time action is concerned with both the plan and subsequent re-evaluations of buffer penetration. By the combination of explicit buffer thresholds and the expediting efficiency index, both of which act as action indicators, the suggested method is able to provide a quick response to real-time schedule deviations as well as to make more effective corrective decisions concerning the project time and cost objectives. In contrast, there is no such consideration of expediting efficiency in the RBMA method. Any buffer consumption that penetrates into the red zone will demand for expediting (Leach, 2005), whereas it might be the case that a duration reduction of some activities has little or no effect on the project completion time, or a huge impact on the cost objective. Therefore, the proposed method is generally more efficient in the project tracking and control process than the conventional buffer management method.

\section{Conclusions}

This article introduces an efficient project control method on the basis of critical chain buffer management for dynamically evaluating and expediting probabilistic projects in a cost-effective manner. The new method was then tested and compared with the predominant buffer management approach and the simulation results prove its promising benefits in almost all aspects, especially in generating significant cost savings. Our contribution is threefold: (1) we incorporate uncertainty into the project expediting decision process and allow dynamic re-evaluations of the project performance on a real-time basis, (2) we develop a simulation-based index that estimates the improvement of TPCP relative to the cost of expediting individual activities, and use the index as a selection criterion to make beneficial crashing decisions, (3) we highlight the ability of the CC/BM methodology to facilitate project managers to implement an effective and comprehensive project management considering time, cost and resource constraints, concurrently.

The study suggests a number of new avenues for future research. To apply the 
method to real projects in order to further testify its effectiveness as well as to explore some of the problems in its practical use will be the first extension. Furthermore, the suggested solution procedure (including the computation of the expediting efficiency index) could be embedded into some existing software to automatically generate effective action guidelines. Another significant area of research would be to consider total project costs (consisting of a project completion time penalty plus crashing and overhead costs) and assign a cost budget to exhibit a more applicable control strategy within the CC/BM framework. We believe that this research has been an effort in the right direction for improving project control decisions to offer an overall time and cost management system. On a larger scale, some production processes that can be organized as a project could employ the proposed principle to expedite certain operations in a cost-effective manner as well as to guarantee an on-time delivery of customer orders.

Acknowledgements This research has been supported by the China Scholarship Council. We would like to acknowledge the China Scholarship Council for the financial support and the Research Center for Operations Management of the KU Leuven for providing a visiting research period to Xuejun $\mathrm{Hu}$. We would also like to thank the editors and the anonymous reviewers for their valuable comments and suggestions, which help to improve this paper.

Funding This work was supported by the National Natural Science Foundation of China under Grant [No. 71271097] and Grant [No. 71201119].

\section{References}

Alfieri, A., Tolio, T., and Urgo, M. 2011. A project scheduling approach to production planning with feeding precedence relations. International Journal of Production Research49(4), 995-1020.

Baker, B.M. 1997. Cost/time tradeoff analysis for the critical path method: A derivation of the network flow approach. Journal of the Operational Research Society 48, 1241-1244.

Bie, L., Cui, N., and Zhang, X. 2012. Buffer sizing approach with dependence assumption 
between activities in critical chain scheduling. International Journal of Production Research 50(24), 7343-7356.

Blackstone Jr, J. H., Cox III, J. F., and Schleier Jr, J. G. 2009. A tutorial on project management from a theory of constraints perspective. International Journal of Production Research 47(24), 7029-7046.

Bregman, R.L. 2009a. A heuristic procedure for solving the dynamic probabilistic project expediting problem. European Journal of Operational Research 192, 125-137.

Bregman R.L. 2009b. Preemptive expediting to improve project due date performance. Journal of the Operational Research Society 60, 120-129.

Bowman, R.A. 2006. Developing activity duration specification limits for effective project control. European Journal of Operational Research 174(2), 585-593.

Büchmann-Slorup, R. 2014. Applying critical chain buffer management theory in location-based management. Construction Management and Economics 32(6), 1-14.

Creemers, S., Demeulemeester, E., and Van de Vonder, S. 2014. A New Approach for Quantitative Risk Analysis.Annals of Operations Research 213, 27-65.

Chen, S. P., and Tsai, M. J. 2011. Time-cost trade-off analysis of project networks in fuzzy environments. European Journal of Operational Research 212(2), 386-397.

Demeulemeester, E., and Herroelen,W. 2002. Project scheduling: A research handbook. Boston: Kluwer Academic Publishers.

Fulkerson, D.R. 1961. A network flow computation for project cost curve. Management Science 7, 167-178.

Goh, J., and Hall, N.G. 2013. Total cost control in project management via satisficing. Management Science59(6), 1354-1372.

Goldratt, E.M. 1997. Critical Chain. New York: The North River Press.

Gutjahr, W. J., Strauss, C., and Wagner, E. 2000. A stochastic branch-and-bound approach to activity crashing in project management. INFORMS Journal on Computing 12(2), $125-135$.

Herroelen, W., and Leus, R. 2001. On the merits and pitfalls of critical chain scheduling. Journal of Operations Management 19(5), 559-577.

Herroelen, W., and Leus, R. 2004. Robust and reactive project scheduling: a review and 
classification of procedures. International Journal of Production Research 42(8), $1599-1620$.

Herroelen, W., and Leus, R. 2005. Project scheduling under uncertainty: Survey and research potentials. European Journal of Operational Research 165, 289-306.

Jiang, A., and Zhu, Y. 2010. A multi-stage approach to time-cost trade-off analysis using mathematical programming. International Journal of Construction Management 10(3), $13-27$.

Johnson, G.A., and Schou, C.D. 1990. Expediting projects in PERT with stochastic time estimates. Project Management Journal 21, 29-33.

Kelley Jr., J.E. 1961. Critical-path planning and scheduling: Mathematical basis. Operations Research 9, 296-320.

Kerzner, H. R. 2013. Project Management: A Systems Approach to Planning,Scheduling, and Controlling (11th ed.). Hoboken, NJ: Wiley.

Klastorin, T. 2004. Project management: tools and trade-offs. Hoboken, NJ: Wiley.

Kolisch, R., and Sprecher, A. 1996. PSPLIB - A project scheduling problem library. European Journal of Operational Research 96, 205-216.

Kuo, T.C., Chang, S.H.,and Huang, S.N.2009. Due-date performance improvement using TOC's aggregated time buffer method at a wafer fabrication factory. Expert Systems with Applications 36(2), 1783-1792.

Leach, L.P. 2005. Critical Chain Project Management (2th ed.). London: Artech House.

Long L. D., and Ohsato A. 2008. Fuzzy critical chain method for project scheduling under resourceconstraintsanduncertainty. InternationalJournalof Project Management 26(6), 688-698.

Mitchell, G., and Klastorin, T. 2007. An effective methodology for the stochastic project compression problem. IIE Transactions 39(10), 957-969.

Newbold, R.C. 1998. Project management in the fast lane - applying the theory of constraints. Boca Raton: The St.Lucie Press.

Papadopoulos, C.T., O’Kelly, M.E.J., and Tsadiras, A.K. 2013. A DSS for the buffer allocation of production lines based on a comparative evaluation of a set of search algorithms. International Journal of Production Research51(14), 4175-4199. 
Peng, W., and Huang, M. 2014. A critical chain project scheduling method based on a differential evolution algorithm. International Journal of Production Research52(13), 3940-3949.

Phillips Jr., S., and Dessouky, M.I. 1977. Solving the project time/cost tradeoff problem using the minimal cut concept. Management Science 24, 393-400.

Rabbani, M., Fatemi Ghomi, S.M.T., Jolai, F., and Lahiji,N.S.2007. A New Heuristic for Resource-Constrained Project Scheduling in Stochastic Networks using Critical Chain Concept. European Journal of Operational Research 176(2), 794-808.

Ricketts, J.A. 2008. Reaching the goal. Westford, MA: IBM Press.

Tian, W., and Demeulemeester, E. 2014. Railway scheduling reduces the expected project makespan over roadrunner scheduling in a multi-mode project scheduling environment. Annals of Operations Research 213(1), 271-291.

Tukel, O.I., Rom, W.O., and Eksioglu, S.D. 2006. An investigation of buffer sizing techniques in critical chain scheduling. European Journal of Operational Research172(2), 401-416.

Turki, S., Hennequin, S., and Sauer, N. 2013. Perturbation analysis for continuous and discrete flow models: a study of the delivery time impact on the optimal buffer level. International Journal of Production Research 51(13), 4011-4044.

Turner, J.R. 2008. The Handbook of Project-based Management. New York: McGraw-Hill.

Umble, M., and Umble, E.J. 2006. Utilizing buffer management to improve performance in a healthcare environment. European Journal of Operational Research 174, 1060-1075.

Van de Vonder, S., Demeulemeester, E., Herroelen W., and Leus, R.2005. The use of buffers in project management: The trade-off between stability and makespan. International Journal of Production Economics 97, 227-240.

Vanhoucke, M. 2012. Project Management with dynamic scheduling. Berlin: Springer.

Van Slyke, R.M. 1963. Letter to the editor - Monte Carlo methods and the PERT problem. Operations Research 11(5), 839-860.

Wang, J. 2005. Constraint-based schedule repair for product development projects with time-limited constraints. International Journal of Production Economics 95, 399-414.

Wu, H.H., Chen, C.P., Tsai, C.H., and Tsai, T.P.2010. A study of an enhanced simulation 
model for TOC supply chain replenishment system under capacity constraint. Expert Systems with Applications 37(9), 6435-6440.

Yuan, K.J., Chang, S.H., and Li, R.K.2003. Enhancement of Theory of Constraints replenishment using a novel generic buffer management procedure. International Journal of Production Research 41(4), 725-740.

Zhu, Y. and Pandit, A. 2007. An ontology-based approach to support decision-making for the design of ETO(Engineer-To-Order) products. Automation in Construction 16(6), 759-770. 